\title{
Page Kidney Phenomenon following Kidney Graft Biopsy: A Medical Emergency
}

\section{Jackie Mak ${ }^{1^{*}}$ and Bulang $\mathrm{He}^{2}$}

${ }^{1}$ General surgical department, Sir Charles Gairdner Hospital, Hospital Avenue, Nedlands 6009, Perth, Australia

${ }^{2}$ Liver and Kidney Transplant Service, Sir Charles Gairdner Hospital, School of Surgery, The University of Western Australia, Nedlands, Perth, WA 6009 Australia

\section{Abstract}

Percutaneous kidney allograft biopsy is commonly used for either diagnostic purpose or as a protocol to identify subclinical pathology. It is a safe procedure with a low incidence of the major complication. Page kidney phenomenon is one of rare complication following the biopsy, which will need urgent surgical intervention. Two cases in our cohort developed Page kidney phenomenon following percutaneous kidney allograft biopsy under US guidance. One case presented to the emergency department 10 days after biopsy with increased pain over the kidney graft, whereas another case developed symptoms during the observation period immediate post the biopsy. Both patients underwent emergency surgical exploration and haematoma evacuation. In the former case, the kidney graft was lost due to extensive haemorrhagic necrosis of the kidney parenchyma, while in the latter case, the kidney graft was salvaged with satisfactory function over 3 years follow up. In conclusion, the Page kidney phenomenon can occur spontaneously, following kidney biopsy or trauma in the setting of kidney transplant. Doppler ultrasound is a very useful modality for the diagnosis. Prompt surgical exploration and evacuation of the haematoma is fundamental in order to salvage the kidney graft.

\section{Introduction}

Percutaneous renal allograft biopsy is commonly performed as a protocol or diagnostic biopsy after kidney transplantation to detect rejection, recurrence of kidney disease, infection and calcineurin inhibitor (CNI) nephrotoxicity [1]. It is generally considered as a safe procedure under ultrasound guidance. However, some complications associated with this procedure can occur. Most common complications are minor and can be managed conservatively. Major complications can occur in about $1 \%$ of cases [2,3], which may require blood transfusion, interventional procedure or even surgical exploration. Page kidney phenomenon is one of the rare but serious complications, in which the renal parenchyma is tamponade and its perfusion is compromised as a result of haematoma formation under the renal capsule. This will lead to deterioration in kidney function and graft loss if the diagnosis and management are delayed [4]. Therefore, early recognition of page kidney phenomenon is crucial as prompt evacuation of the haematoma can prevent graft loss and allow complete recovery of renal function [5]. Here we reported two cases of page phenomenon after kidney graft biopsy in our institution. The literatures were also reviewed.

\section{Case 1}

A 62-year-old lady with end stage kidney disease due to Type 2 Diabetes underwent second renal transplant. On day 4 post transplantation, she developed antibody mediated rejection and was treated per protocol. Two months later, her creatinine remained high around $200 \mathrm{umol} / \mathrm{L}$. A repeat kidney graft biopsy was performed under ultrasound guidance with a 16-gauge needle. The resistive index (RI) of renal arterial branch was 0.72 on Doppler ultrasound prior to kidney biopsy. There was no immediate post biopsy complication and her blood pressure remained stable at $140 / 80 \mathrm{mmHg}$ during the observation period. Her vital signs had been stable as well for six hours during the routine observation. She had no haematuria after biopsy and thus she was discharged home as per the protocol of kidney graft biopsy. Ten days later, she presented to the emergency department with pain over transplanted kidney at left iliac fossa, nausea and vomiting. On examination, she looked stable and had a blood pressure of $138 / 86 \mathrm{mmHg}$. There was tenderness over the kidney graft site. Her haemoglobin was $103 \mathrm{~g} / \mathrm{L}$ compared to $107 \mathrm{~g} / \mathrm{L}$ on the day of her last kidney biopsy, creatinine was elevated to 334 $\mu \mathrm{mol} / \mathrm{L}$ from $200 \mu \mathrm{mol} / \mathrm{L}$. Doppler ultrasound showed a haematoma in the interpolar region of the transplanted kidney. There was no vascular flow demonstrated within this area. The vascular waveforms were abnormal with absence of diastolic flow in the upper and lower pole of the transplanted kidney. The RI was increased to 1.2 (Figure 1 and Figure 2). CT of the abdomen revealed a subcapsular haematoma in the transplanted kidney (Figure 3). The diagnosis was confirmed as page kidney phenomenon. The patient underwent urgent surgical exploration and evacuation of the subcapsular haematoma in the transplanted kidney. There was no active bleeding identified during exploration. The intraoperative Doppler US showed some recovery of kidney perfusion. However, her kidney graft function continued to deteriorate postoperatively. On day 1 post operation, Doppler ultrasound demonstrated normalization of vascular traces in upper and lower pole of kidney graft, but no flow could be obtained in the inter polar region. On day 2 post-operation, renal nuclear perfusionscan showed absence of perfusion and function of the kidney graft. The decision was made to remove the transplanted kidney.

"Corresponding Author: Dr. Bulang $\mathrm{He}$, Liver and Kidney Transplant Service, Sir Charles Gairdner Hospital, School of Surgery, The University of Western Australia, Nedlands, Perth, WA 6009, Australia; Email: bulang.he@health.wa.gov.au

Citation: Mak J, He B (2017) Page Kidney Phenomenon following Kidney Graft Biopsy: A Medical Emergency. Int J Surg Surgical Porced 2: 127. https://doi. org/10.15344/2456-4443/2017/127

Copyright: @ 2017 Mak et al. This is an open-access article distributed under the terms of the Creative Commons Attribution License, which permits unrestricted use, distribution, and reproduction in any medium, provided the original author and source are credited. 
Citation: Mak J, He B (2017) Page Kidney Phenomenon following Kidney Graft Biopsy: A Medical Emergency. Int J Surg Surgical Porced 2: 127. https://doi. org/10.15344/2456-4443/2017/127

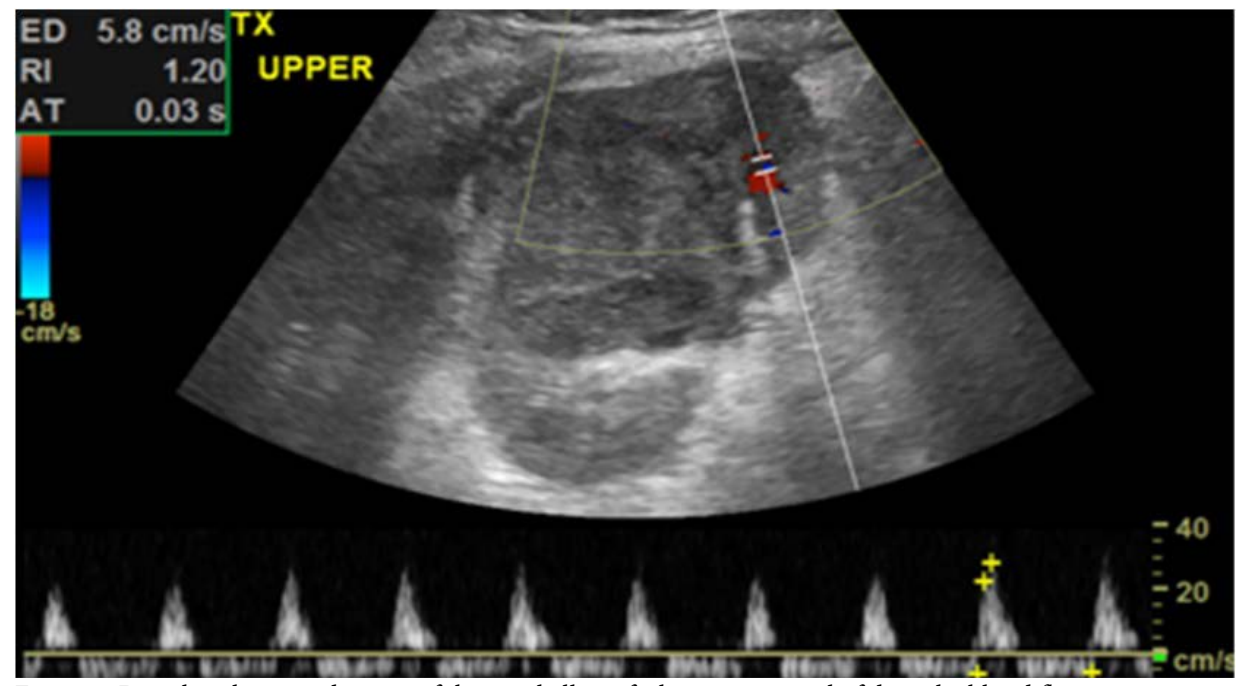

Figure 1: Doppler ultrasound image of the renal allograft showing reversal of diastolic blood flow in upper pole of transplanted kidney.

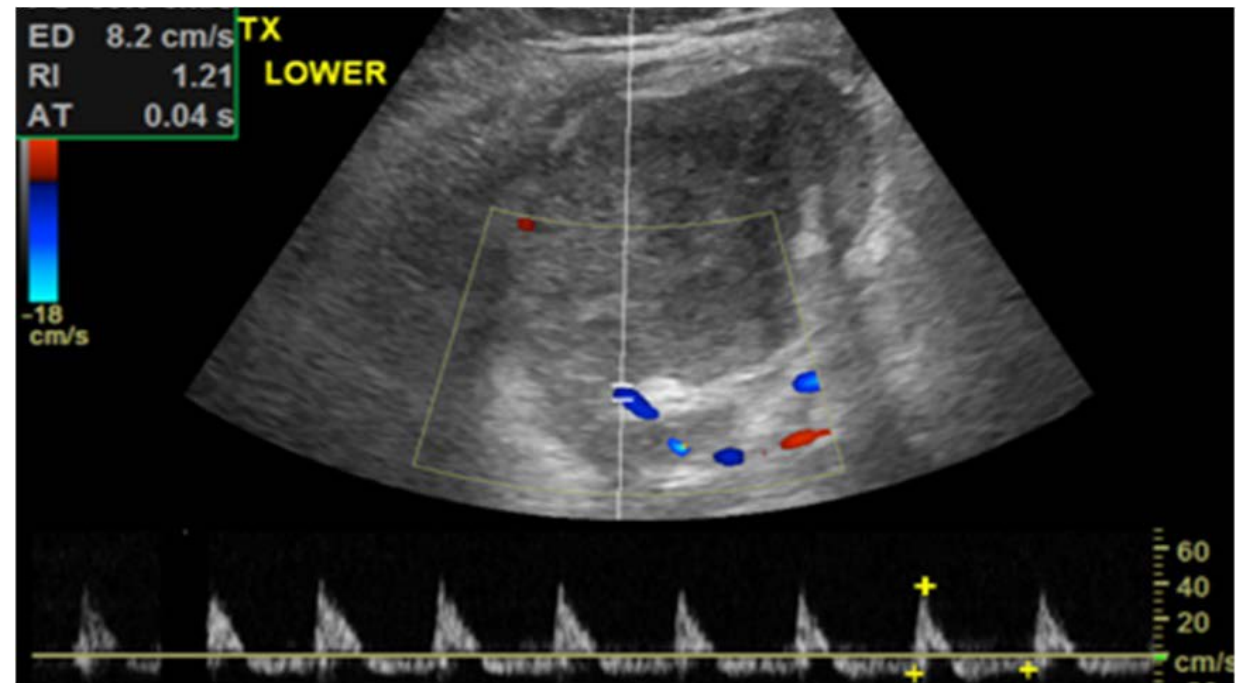

Figure 2: Doppler ultrasound image of the renal allograft showing reversal of diastolic blood flow in lower pole of transplanted kidney

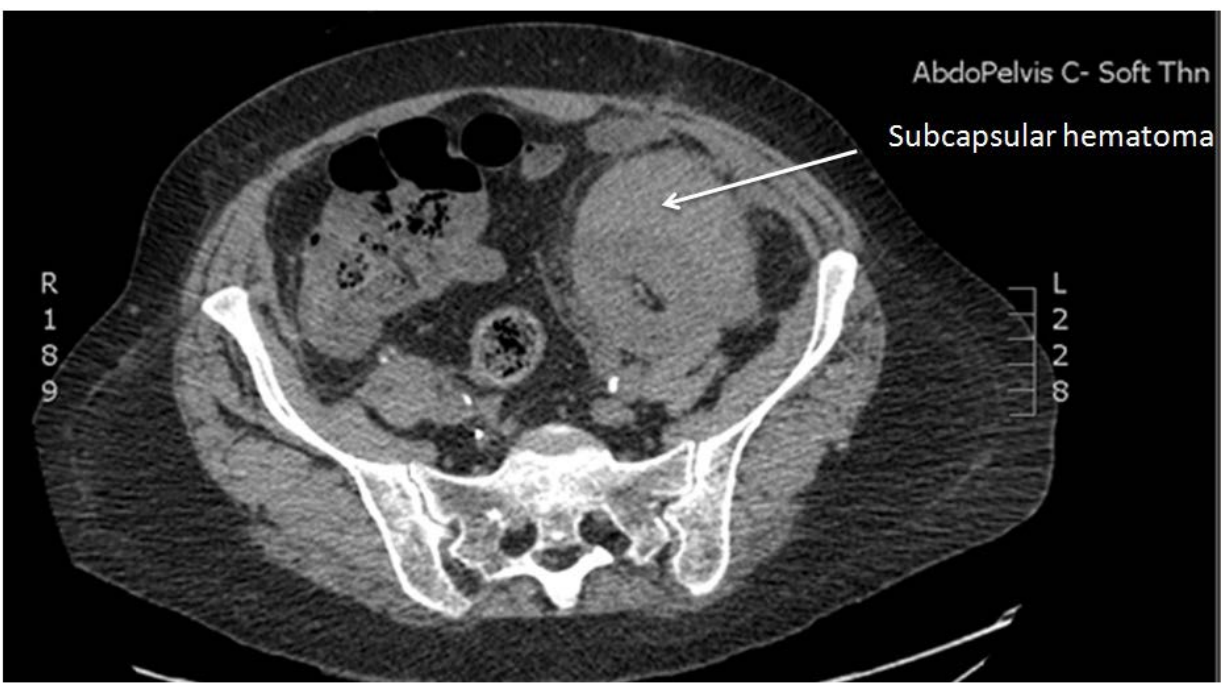

Figure 3: CT image showing subcapsular haematoma in the transplanted kidney. 
Histopathology confirmed that the kidney graft was non-viable with extensive haemorrhagic infarction involving over $90 \%$ of the renal parenchyma.

\section{Case 2}

A 58-year-old lady underwent an uncomplicated renal transplantation for end-stage renal failure secondary to polycystic kidney disease. Her creatinine on discharge was $97 \mathrm{umol} / \mathrm{L}$. Three months post transplantation, she underwent protocol biopsy.

The patient had no coagulopathy. Biopsy was performed under ultrasound guidance with a 16-gauge needle. The RI of renal arterial branch was 0.66 before biopsy. There was no immediate complication and her blood pressure was 130/60 $\mathrm{mmHg}$ immediately after biopsy.

During the observation period post biopsy, she subsequently developed macroscopic haematuria and worsening pain over transplanted kidney graft at right illiac fossa. She then became oligouric six hours post biopsy and her blood pressure had gone up to $160 / 100 \mathrm{mmHg}$. On repeat blood test, serum creatinine was increased to $182 \mathrm{umol} / \mathrm{L}$ from $98 \mathrm{umol} / \mathrm{L}$ prior to biopsy and her haemoglobin level was stable. An urgent Doppler ultrasound was performed. There was a subcapsular haematoma in the inferior pole of the transplanted kidney with a high resistivity index of 1 in the interlobar arteries of the kidney parenchyma. There was also a reversal diastolic blood flow (Figure 4 and Figure 5). The decision was made for urgent exploration and evacuation of the subcapsular haematoma. During surgery a subcapsular haemotoma was seen, which was evacuated. Following that an active bleeding source was identified from the biopsy puncture site at the lower pole of the kidney. The haemostasis was achieved by oversewing the bleeder with a $3 / 0$ Vicryal suture.

The patient recovered well with good urine output post-surgical exploration. On repeat Doppler US, there was a normalised parenchymal blood flow in the transplanted kidney (Figure 6). However, the kidney graft function had continuously worsened over the next few days with a peak $\mathrm{Cr}$ level of $744 \mu \mathrm{mol} / \mathrm{L}$ on day 6 post surgery. Fortunately, the $\mathrm{Cr}$ level started to improve over the next few weeks. It was at $209 \mu \mathrm{mol} / \mathrm{L}$ one month post-surgery and slowly recovered to a baseline of $120 \mu \mathrm{mol} / \mathrm{L}$. The kidney graft function was stable over the 3 years follow up.

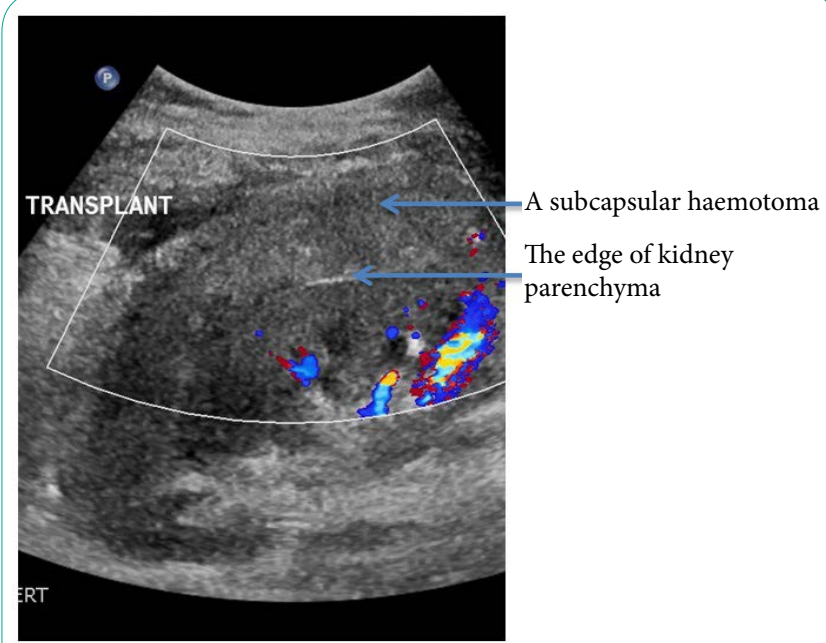

Figure 4: A subcapsular haematoma on Ultrasound.

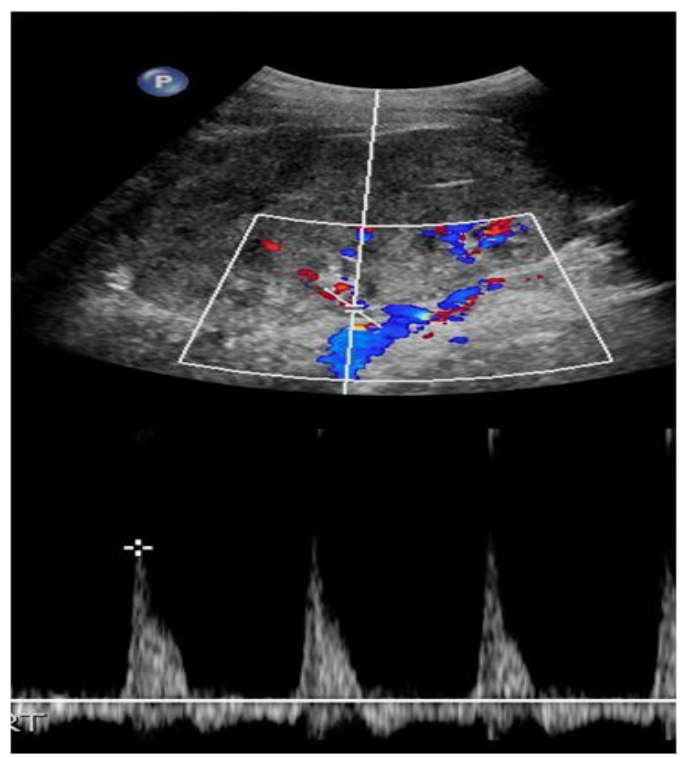

Figure 5: Doppler Ultrasound showed absence of diastolic flow.

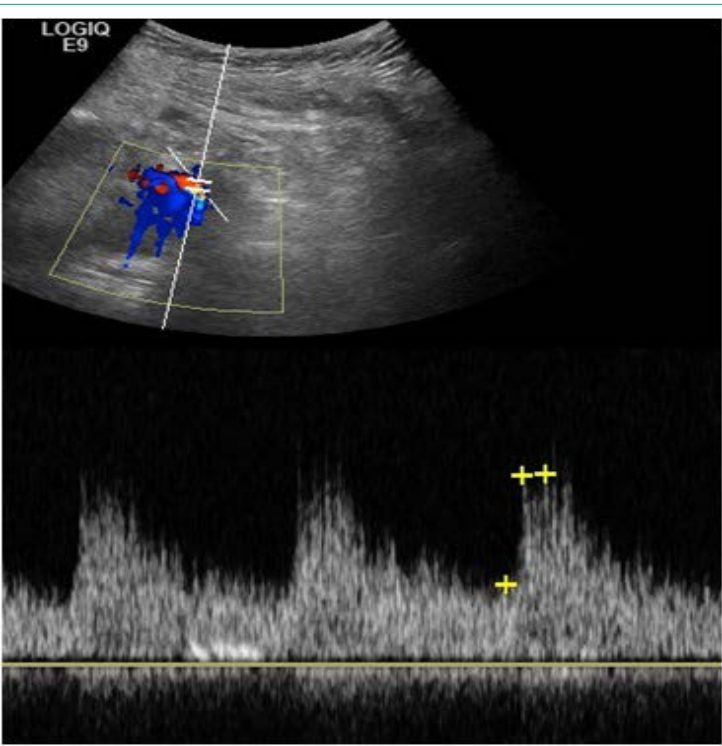

Figure 6: Doppler Ultrasound showed normal waveforms of renal parenchyma.

\section{Discussion}

Percutaneous renal biopsy has been increasingly used as a protocol biopsy after kidney transplantation. It is also commonly performed to diagnose the underlying pathology when the renal function deteriorates. However, it is associated with some potential complications such as haematuria, urinary tract obstruction by clots, formation of pseudoaneurysm and/or arteriovenous fistula and perigraft or subcapsular haematoma. The incidence of the complications ranged from $0.7 \%$ to $30 \%$ [5], whereas the major complication requiring interventional procedure was about $1 \%$ [4].

The Page kidney phenomenon was first described by Dr. Page in an experimental model in 1939 [6]. In the experiment, cellophane was wrapped around a native kidney leading to the constriction in renal parenchyma and hypertension [6]. In the context of kidney transplantation, Page kidney phenomenon can occur spontaneously 
Page 4 of 4

after kidney transplantation 7 , after percutaneous kidney graft biopsy $[4,8-10]$, or after trauma to the allograft [11]. It can also occur in a native kidney. There is a paucity of reports describing subcapsular haematoma following kidney graft biopsy $[4,7-10,12,13]$. It has the same effect as demonstrated in the Page kidney experiment. The haematoma compresses on the renal parenchyma as a tamponade effect and thus reduces the kidney perfusion. Wanic-Kossowska et al . [12] reported 3 cases of Page kidney phenomenon in consecutive 800 renal biopsies; two of cases developed subcapsular haematoma and one developed a huge peri-kidney haematoma. In addition, Chung et al. [10] reported 4 cases of Page kidney phenomenon in 518 renal transplant biopsies. The page kidney phenomenon may develop almost immediately after percutaneous renal biopsy. However, it could have delay presentation sometimes such as 10 days following biopsy [11], as in our case 1 . The late presentation could be due to the rupture of a pseudpaneurysm that was formed following percutaneous biopsy".

Given the rarity of page kidney phenomenon, its diagnosis can be easily missed. In our opinion, an acute pain over the graft with reduction in urine output is an alert for suspicion of Page kidney phenomenon after percutaneous biopsy. Urgent investigation should be performed with Doppler US of the kidney graft and blood test for Cr level. It was also suggested that elevation in blood pressure is the hallmark of presentation due to the activation of renin-angiotensin system 10, 1314. In our experience, Doppler US is a useful modality for prompt diagnosis in the setting of percutaneous kidney graft biopsy. The absence or reversal of diastolic blood flow with elevated RI is a specific feature of Page kidney phenomenon although differential diagnoses also include acute tubular necrosis, acute rejection and renal vein thrombosis 2 . Repeat imaging is not of extra diagnostic value, as the time to surgical exploration is critical. However, CT angiogram may be required for further investigation for active bleeder, arterial-venous fistula or pesudoaneurysm formation, which would provide further information for interventional management such as selective embolization and so forth. The haemoglobin drop may not be very obvious as a small amount of haematoma can have a significant tamponade effect to kidney graft perfusion. Therefore, in our opinion, prompt surgical evacuation of the hematoma is fundamental in the setting of kidney transplant if the kidney perfusion is compromised. Any delay of diagnosis and surgical intervention may result in kidney graft loss as in our case 1 . This is because the renal graft capsule is usually thickened and lack of elasticity to allow expansion of haemotoma. As a result, the kidney graft perfusion is compromised. It was understood that the treatment options for page kidney phenomenon include conservative observation allowing the haematoma to be absorbed; interventional drainage and surgical intervention if it occurred in the native kidney. However, in the setting of renal transplantation, open surgical exploration and evacuation of the haematoma is preferred as percutaneous drainage may not resolve the tamponade effect completely, resulting in the recurrence of Page kidney phenomenon and loss of kidney graft 15. Radiological intervention is a minimally invasive approach if the kidney graft circulation is not compromised.

In conclusion, Page kidney phenomenon is a rare but serious complication after renal graft biopsy. It should be considered as medical emergency. The patient should be fully informed about the symptoms of Page kidney phenomenon when subject to percutaneous kidney graft biopsy. Doppler US is an acute useful modality for prompt diagnosis of Page kidney phenomenon. The early recognition of Page kidney phenomenon and prompt surgical intervention is fundamental in order to prevent the graft loss.

\section{Conflict of interest}

No authors have a conflict of interest or any financial tie to disclose.

\section{Author's Contributions}

Jackie Mak: Conducted data collection, literature review and wrote the paper.

Bulang He: Supervised the study and revised the paper.

\section{References}

1. Tanabe $\mathrm{T}$ (2014) The value of long-term protocol biopsies after kidney transplantation. Nephrology(Carlton) 19: 2-5.

2. Adjei-Gyamfi Y, Koffman G, Amies T, Easty M, Marks SD, et al. (2014) Reversible acute anuric kidney injury after surgical evacuation of perinephric hematomas as a complication of renal transplant biopsies. Pediatric Transplantation 18: E262-E265.

3. Ahmad I (2004) Biopsy of the transplanted kidney. Semin Intervent Radiol 21: $275-281$.

4. Maurya KK, Bhat HS, Mathew G, Kumar G (2011) Page kidney following renal allograft biopsy - early recognition and treatment. Saudi J Kidney Dis Transpl 22: 1012-1013.

5. Heffernan E, Zwirewich C, Harris A, Nguan C (2009) Page Kidney after Renal Allograft Biopsy: Sonographic Findings. J Clin Ultrasound 37: 226229.

6. Page $\mathrm{IH}$ (1939) The production of persistent arterial hypertension by cellophane perinephritis. JAMA 113: 2046-2048.

7. Butt FK, Seawright AH, Kokko KE, Hawxby AM (2010) An unusual presentation of a Page kidney 24 days after transplantation: case report. Transplant Proc 42: 4291-4294.

8. Haydar A, Bakri RS, Prime M, Goldsmith DJ (2003) Page kidney--a review of the literature. J Nephrol 16: 329-333.

9. Kliewer M, Carroll B (1991) Ultrasound of the day. Radiographics 11: 336337.

10. Chung J, Caumartin $Y$, Warren J, Luke $P$ (2008) Acute page kidney following renal allograft biopsy: A complication requiring early recognition and treatment. AmJ Transplant 8: 1323-1328.

11. Lauren E (2017) Malinzak Allograft loss from acute Page kidney secondary to trauma after kidney transplantation. World J Transplant 7: 88-93.

12. Wanic-Kossowska M, Kobelski M, Oko A (2005) Arterial hypertension due to perirenal and subcapsular hematoma induced by renal percutaneous biopsy. Int Urol Nephrol 37: 141-143.

13. Morgan TA, Chandran S, Burger IM, Zhang CA, Goldstein RB (2016) Complications of Ultrasound-Guided Renal Transplant Biopsies. Am J of Transplantation 16: 1298-1305.

14. Gibney EM, Edelstein CL, Wiseman AC, Thomas B (2005) Page kidney causing reversible acute renal failure: an unusual complication of transplant biopsy. Transplantation 80: 285-286.

15. Kapoor R, Zayas C. Mulloy L, Jagadeesan M (2016) Recurrence of acute page kidney in a renal transplant allograft. Case Rep Med 2016: 3898307. 